Proyecciones Journal of Mathematics

Vol. 29, No 3, pp. 165-182, December 2010.

Universidad Católica del Norte

Antofagasta - Chile

\title{
ON THE DISTRIBUTIONS OF THE DENSITIES INVOLVING NON-ZERO ZEROS OF BESSEL AND LEGENDRE FUNCTIONS AND THEIR INFINITE DIVISIBILITY
}

\author{
HEMANT KUMAR \\ D. A-V. P. G. COLLEGE KANPUR, INDIA \\ M. A. PATHAN \\ UNIVERSITY OF BOTSWANA, BOTSWANA \\ and \\ R. C. SINGH CHANDEL \\ D. V. P. G. COLLEGE ORAI, INDIA \\ Received: September 2009. Accepted : January 2010
}

\begin{abstract}
In the present paper, we introduce the probability density functions involving non-zero zeros of the Bessel and Legendre functions. Then, we evaluate the distributions of the characteristic functions defined by these probability density functions and again obtain their related functions and polynomials. Finally, we prove the infinite divisibility of these probability density functions.
\end{abstract}

2000 Mathematics Subject Classification : 33C20, 62E15, 60E05, $60 E 10$.

Keywords and Phrases : Probability density functions, characteristic functions, non zero zeros of Bessel and Legendre functions, infinite divisibility. 


\section{Introduction}

Hochstadt [4] has derived the infinite product representation of the Bessel function of order real $v$, such that

$$
J_{v}(z)=\frac{\left(\frac{z}{2}\right)^{v}}{\Gamma(v+1)} \prod_{k=1}^{\infty}\left(1-\frac{z^{2}}{z_{k}^{2}}\right), z \in C, z_{k} \neq 0, \forall k=1,2, \ldots
$$

as considering the sequence of integrals

$$
I_{n}^{(1)}=\frac{1}{2 \pi i} \int_{C_{n}} \frac{F_{1}(\xi)}{\xi(\xi-z)} d \xi, i=\sqrt{(-1)}, n=1,2, \ldots
$$

where, the sequence of circles $\left\{C_{n}\right\}$ about the origin such that $C_{n}$ includes all non-zero real zeros, $\pm z_{1}, \ldots, \pm z_{n}$, of Bessel function $J_{v}(z)$, the point $z$ such that for fixed $z \neq z_{n}$ and not passing through any zeros, also, $\mathrm{F}_{1}(z)=\frac{G^{\prime}(z)}{G(z)}, G(z)=z^{-v} J_{v}(z), \forall z \in \mathbf{C}$ (the set of complex numbers).

Again, Kumar and Srivastava [6] have obtained the infinite product representation of the Legendre function such that

$$
P_{\mu}(z)=z^{\mu-2} \prod_{k=1}^{\infty}\left(1-\frac{1-z^{2}}{1-\varsigma_{k}^{2}}\right), z \neq 0, z \in \mathbf{C}
$$

and $\varsigma_{k} \neq 0$, and $-1<\varsigma_{k}<1, \forall k=1,2 \ldots$

by considering the sequence of integrals

$$
I_{n}^{(2)}=\frac{1}{2 \pi i} \int_{C_{n}} \frac{F_{2}(t)}{(t-z)} d t, i=\sqrt{(-1)}, n=1,2, \ldots
$$

where, the sequence of circles $C_{n}$ about the origin such that $C_{n}$ includes all non-zero and real zeros, $\left.\pm \varsigma_{1}, \ldots, \pm \varsigma_{(} n,\right)$ lie between -1 to +1 (i.e. $-1<$ $\left.\varsigma_{k}<1, \forall k=1,2, \ldots, n\right)$ of Legendre polynomial of degree $\mu$, the point $z$ twice such that for fixed $z \neq \varsigma_{n}$ and not passes through any zeros. Also, it has $F_{2}(z)=\frac{\left.H^{(} z\right)}{H(z)}, H(z)=z^{-\mu} P_{\mu}(z), z \neq 0$ and $z \in \mathbf{C}$.

The concept of infinite divisibility and the decomposition of distributions arise in probability and statistics in relation to seeking families of probability distributions that might be a natural choice in certain applications, in the same way that the normal distribution is. The term infinitely 
divisible characteristic function is used for the characteristic function of any infinitely divisible distribution. These distributions play a very important role in probability theory in the context of limit theorems. Takano [10] has conjectured that following probability density in normed conjugate product of Gamma functions

$$
\frac{2}{\pi} \Gamma(1-i x) \Gamma(1+i x)=\frac{2 i x}{\sin \pi i x}=\frac{2}{\pi} \frac{1}{\prod_{k=1}^{\infty}\left(1+\frac{x^{2}}{k^{2}}\right)},-\infty<x<\infty
$$

is infinite divisible.

Several authors studied infinite divisibility of many functions and their related topics (See, Bondesson [2], Goovaerts, D Hooge and Pril [3], Kelkar [5], Steutel [8], Takano [9, 10] and Thorin [11] etc.). Motivated by the above work and from (1.1), (1.3) and (1.5), we introduce following equalities in the form of probability density functions:

$$
\frac{(i x)^{v}}{2^{v} \Gamma(v+1) J_{v}(i x)}=\frac{1}{\prod_{k=1}^{\infty}\left(1+\frac{x^{2}}{z_{k}^{2}}\right)},-\infty<x<\infty
$$

and

$$
\frac{(i x)^{\mu}}{x^{2} P_{\mu}(i x)}=\frac{1}{\prod_{k=1}^{\infty}\left(\frac{x^{2}+\varsigma_{k}^{2}}{1-\varsigma_{k}^{2}}\right)},-\infty<x<\infty
$$

respectively, and where, $z_{k} \neq 0$ and $\varsigma_{k} \neq 0-4$, and $-1<\varsigma_{k}<1$, $\forall k=1,2 \ldots, n$, are zeros of Bessel and Legendre functions, respectively. Here, we conjecture that above densities (1.6) and (1.7) are infinitely divisible. Therefore, for that we define following probability density functions:

$$
f_{1}(x)=\lambda_{1} \frac{\prod_{k=1}^{n}\left(z_{k}^{2}\right)}{\prod_{k=1}^{n}\left(x^{2}+z_{k}^{2}\right)},-\infty<x<\infty
$$

and

$$
f_{2}(x)=\lambda_{2} \frac{\prod_{k=1}^{n}\left(1-\varsigma_{k}^{2}\right)}{\prod_{k=1}^{n}\left(x^{2}+\varsigma_{k}^{2}\right)},-\infty<x<\infty
$$


where, $z_{k} \neq 0$ and $\varsigma_{k} \neq 0$, and $-1<\varsigma_{k}<1, \forall k=1,2, \ldots, n$, are zeros of Bessel and Legendre functions, respectively and $\lambda_{1}$ and $\lambda_{2}$ are arbitrary constants.

\section{The Distributions of the Characteristic Function Involving Non-zero Zeros of Bessel Function and its Related Poly- nomials}

Theorem-1 : If in the complex upper half plane for the probability density function (1.8) there exists a characteristic function

$$
\Phi_{1}(t)=\int_{-\infty}^{\infty} e^{i t x} \frac{\lambda_{1} \prod_{k=1}^{n}\left(z_{k}^{2}\right)}{\prod_{k=1}^{n}\left(x^{2}+z_{k}^{2}\right)} d x,-\infty<t<\infty
$$

then, there holds the distribution formula of non-zero real zeros, $z_{k}$, (i.e. $z_{k} \neq 0$ ), $\forall k=1,2, \ldots, n$, of Bessel function on the real curve of $\mathrm{t}$ such that

$$
\Phi_{1}(t)=\pi \lambda_{1} \sum_{k=1}^{n} z_{k} \frac{\prod_{l=1, l \neq k}^{n}\left(z_{l}^{2}\right) \exp \left[-z_{k}|t|\right]}{\prod_{l=1, l \neq k}^{n}\left(-z_{k}^{2}+z_{l}^{2}\right)},-\infty<t<\infty
$$

Proof : In the complex plane, consider the contour integral

$$
\int_{C} e^{i t z} \frac{\lambda_{1} \prod_{k=1}^{n}\left(z_{k}^{2}\right)}{\prod_{k=1}^{n}\left(z^{2}+z_{k}^{2}\right)} d z,--\infty<t<\infty
$$

where, $C$ is the closed contour consisting of $\gamma$, the upper half of the large circle $|z|=R$, the real axis from $-R$ to $R$. The poles of the integrand $e^{i t z} \frac{\lambda_{1} \prod_{k=1}^{n}\left(z_{k}^{2}\right)}{\prod_{k=1}^{n}\left(z^{2}+z_{k}^{2}\right)}$ are at $z= \pm i z_{k}, \forall k=1,2 \ldots, n$ and $-\infty<t<\infty$. Also, inside $C$ it has the simple poles at $z=i z_{k},=1,2 \ldots, n$ in the upper half plane and $\lim _{|z| \rightarrow \infty} \frac{\lambda_{1} \prod_{k=1}^{n}\left(z_{k}^{2}\right)}{\prod_{k=1}^{n}\left(z^{2}+z_{k}^{2}\right)}=0$ Hence by Cauchys residue theorem and due to Jordan lemma, we find

$$
\int_{-\infty}^{\infty} e^{i t x} \frac{\lambda_{1} \prod_{k=1}^{n}\left(z_{k}^{2}\right)}{\prod_{k=1}^{n}\left(x^{2}+z_{k}^{2}\right)} d x=\pi \lambda_{1} \sum_{k=1}^{n} z_{k} \frac{\prod_{l=1, l \neq k}^{n}\left(z_{l}^{2}\right) \exp \left[-z_{k}|t|\right]}{\prod_{l=1, l \neq k}^{n}\left(-z_{k}^{2}+z_{l}^{2}\right)}
$$

when $t>0$. 
In the similar way we have

$$
\int_{-\infty}^{\infty} e^{i t x} \frac{\lambda_{1} \prod_{k=1}^{n}\left(z_{k}^{2}\right)}{\prod_{k=1}^{n}\left(x^{2}+z_{k}^{2}\right)} d x=\pi \lambda_{1} \sum_{k=1}^{n} z_{k} \frac{\prod_{l=1, l \neq k}^{n}\left(z_{l}^{2}\right) \exp \left[z_{k} t\right]}{\prod_{l=1, l \neq k}^{n}\left(-z_{k}^{2}+z_{l}^{2}\right)},
$$

when $t<0$.

$$
\int_{-\infty}^{\infty} e^{i t x} \frac{\lambda_{1} \prod_{k=1}^{n}\left(z_{k}^{2}\right)}{\prod_{k=1}^{n}\left(x^{2}+z_{k}^{2}\right)} d x=\pi \lambda_{1} \sum_{k=1}^{n} z_{k} \frac{\prod_{l=1, l \neq k}^{n}\left(z_{l}^{2}\right)}{\prod_{l=1, l \neq k}^{n}\left(-z_{k}^{2}+z_{l}^{2}\right)},
$$

when $t=0$.

Therefore, on combining the equations $(2.4 \mathrm{a}),(2.4 \mathrm{~b})$ and $(2.4 \mathrm{c})$ we evaluate

$$
\begin{aligned}
& \int_{-\infty}^{\infty} e^{i t x} \frac{\lambda_{1} \prod_{k=1}^{n}\left(z_{k}^{2}\right)}{\prod_{k=1}^{n}\left(x^{2}+z_{k}^{2}\right)} d x=\pi \lambda_{1} \sum_{k=1}^{n} z_{k} \frac{\prod_{l=1, l \neq k}^{n}\left(z_{l}^{2}\right) \exp \left[z_{k}|t|\right]}{\prod_{l=1, l \neq k}^{n}\left(-z_{k}^{2}+z_{l}^{2}\right)}, \\
& -\infty<t<\infty
\end{aligned}
$$

Hence, the theorem has been proved.

\subsection{Related Functions and Polynomials:}

Set $u=e^{-|t|}$ (i.e. $\left.|t|=\log u^{-1}\right), 0<u<1$ in $(2.2)$, we find a function

$$
\Phi_{1}(u)=\pi \lambda_{1} u \sum_{k=1}^{n} z_{k} \frac{\prod_{l=1, l \neq k}^{n}\left(z_{l}^{2}\right)(u)^{z} k^{-1}}{\prod_{l=1, l \neq k}^{n}\left(-z_{k}^{2}+z_{l}^{2}\right)} .
$$

Again, set $\pi \lambda_{1} u=\frac{(-1)^{n-1} z_{n} \prod_{l=1}^{n-1}\left(-z_{n}^{2}+z_{l}^{2}\right)}{\prod_{l=1}^{n} z_{l}}$ in (2.6), we find another function

$$
Q_{1}(u)=\frac{(-1)^{n-1} z_{n} \prod_{l=1}^{n-1}\left(-z_{n}^{2}+z_{l}^{2}\right)}{\prod_{l=1}^{n} z_{l}} \sum_{k=1}^{n} z_{k} \frac{\prod_{l=1, l \neq k}^{n}\left(z_{l}^{2}\right)(u)^{z_{k}^{-1}}}{\prod_{l=1, l \neq k}^{n}\left(-z_{k}^{2}+z_{l}^{2}\right)}
$$


Now, set $z_{l}=l, l=1,2, \ldots, n$, in $(2.7)$, we find a polynomial such that

$$
Q_{1}^{(n-1)}(u)=\sum_{k=1}^{n} \frac{(n-1) !(2 n) !}{(n-k) !(n+k) !} k(-u)^{k-1} .
$$

For the probability density (1.5) the Takanos polynomial [10] is given by

$$
P_{n-1}(u)=\sum_{k=1}^{n} \frac{(2 n) !}{(n-k) !(n+k) !} \frac{k}{n}(-u)^{k-1}, n \geq 1 .
$$

Then, make an appeal to the eqns. (2.8) and (2.9) to get a relation

$$
Q_{1}^{(n-1)}(u)=n ! P_{(n-1)}(u), n \geq 1 .
$$

Thus due to the relation (2.10) and the analysis given in [10, section-2, eqns. (2.3)-(2.8)] we may guess the curve of the trigonometric sum which has non- zero zeros (in form of natural numbers) of Bessel function.

Further, set $\pi \lambda_{1} u=\frac{(-1)^{n-1} z_{n} \prod_{l=1}^{n-1}\left(-z_{n}^{2}+z_{l}^{2}\right)}{\prod_{l=1}^{n} z_{l}^{2}}$ in (2.6) and then put $z_{l}=\frac{1}{l}, \forall l=1,2, \ldots, n$, to find a function

$$
R_{1}^{(n-1)}(u)=\sum_{k=1}^{n} \frac{(k)^{2 n-1}}{(n)^{2 n-1}} \frac{(2 n) !}{(n-k) !(n+k) !}\left(-u_{k}\right)^{k-1}, \text { where, }
$$

$\mathrm{u}_{k}=u^{(-1 / k)}, 0<u<1, n \geq 1$.

The proof of (2.11) follows readily from the formula (2.6) by setting $\pi \lambda_{1} u=\frac{(-1)^{n-1} z_{n} \prod_{l=1}^{n-1}\left(-z_{n}^{2}+z_{l}^{2}\right)}{\prod_{l=1}^{n} z_{l}^{2}}$

and then putting $z_{l}=\frac{1}{l}, \forall l=1,2, \ldots, n$, to get

$R_{1}^{(n-1)}(u)=(-1)^{n-1} \prod_{l=1}^{n-1}\left(\frac{n^{2}-l^{2}}{n^{2}}\right) \sum_{k=1}^{n}\left(\frac{n}{k}\right) \prod_{l=1, l \neq k}^{n}\left(\frac{k^{2}}{k^{2}-l^{2}}\right)(u)^{1 / k^{-1}}$

Then from the equation (2.12), we easily obtain (2.11).

If we suppose that 


$$
S_{1}^{(n-1)}(u)=u R_{1}^{((n-1)}(u), S_{1}^{((n-1)}(u) \text { is many times differentiable, }
$$

$\mathrm{n} \geq 1$,

and

$$
H_{n-1}(u)=\sum_{k=1}^{n} \frac{(2 n) !(-1)^{k-1}}{(n-k) !(n+k) !}\left(\frac{k}{n}\right)(u)^{\frac{1}{k}}, n \geq 1
$$

Then with the aid of (2.11), (2.13) and (2.14), we find that

$$
\left(u \frac{d}{d u}\right)^{2 n-2} S_{1}^{(n-1)}(u)=\frac{1}{(n)^{2 n-2}} H_{n-1}(u), n \geq 1 .
$$

Thus due to the relation (2.15) and the analysis given in [10, section-2, eqns. (2.3)-(2.8)] we may guess the curve of the trigonometric sum, this curve has non-zero zeros (in form of rational numbers) of Bessel function.

\section{The Distributions of the Characteristic Function Involv- ing Non-zero Zeros of Legendre Function and its Related Polynomials}

Theorem-2 : If in the complex upper half plane the characteristic function for the density function (1.9) exists by the formula

$$
\Phi_{2}(t)=\int_{-\infty}^{\infty} e^{i t x} \frac{\lambda_{2} \prod_{k=1}^{n}\left(1-\varsigma_{k}^{2}\right)}{\prod_{k=1}^{n}\left(x^{2}+\varsigma_{k}^{2}\right)} d x,-\infty<t<\infty,
$$

then, there holds the distribution formula of non-zero real zeros lying between -1 to $+1, \varsigma_{k},\left(i . e . \varsigma_{k} \neq 0\right.$, and $\left.-1<\varsigma_{k}<1\right), \forall k=1,2 \ldots, n$, of Legendre function on the real curve of $t$ such that

$$
\Phi_{2}(t)=\pi \lambda_{2} \sum_{k=1}^{n}\left(\varsigma_{k}^{-1}-\varsigma_{k}\right) \frac{\prod_{l=1, l \neq k}^{n}\left(1-\varsigma_{k}^{2}\right) \exp \left[-\varsigma_{k}|t|\right]}{\prod_{l=1, l \neq k}^{n}\left(-\varsigma_{k}^{2}+\varsigma_{l}^{2}\right)},-\infty<t<\infty,
$$

Proof : In the similar manner, as analyzed to prove theorem-1, we prove theorem- 2 . 


\subsection{Related Functions and Polynomials:}

Set $u=e^{-|t|}$ (i.e. $|t|=\log u^{-1}, 0<u<1$ in (3.2), to find a function

$$
\Phi_{2}(u)=\pi \lambda_{2} u \sum_{k=1}^{n}\left(\varsigma_{k}^{-1}-\varsigma_{k}\right) \frac{\prod_{l=1, l \neq k}^{n}\left(1-\varsigma_{l}^{2}\right)(u)^{\varsigma_{k}-1}}{\prod_{l=1, l \neq k}^{n}\left(-\varsigma_{k}^{2}+\varsigma_{l}^{2}\right)}, \varsigma_{k} \neq 0
$$

and $-1<\varsigma_{k}<1, \forall k=1,2 \ldots$

$$
\begin{aligned}
& \text { Again, set } \pi \lambda_{2} u=\frac{(-1)^{n-1} \prod_{l=1}^{n-1}\left(\varsigma_{l}^{2}-\varsigma_{n}^{2}\right)}{\prod_{l=1}^{n}\left(1-\varsigma_{l}\right)} \text { in }(3.3), \text { to find a function } \\
& Q_{2}(u)=(-1)^{n-1} \prod_{l=1}^{n-1}\left(\varsigma_{l}^{2}-\varsigma_{n}^{2}\right) \sum_{k=1}^{n} \frac{1}{\varsigma_{k}} \frac{\prod_{l=1}^{n}\left(1+\varsigma_{l}\right)(u)^{\varsigma_{k}-1}}{\prod_{l=1, l \neq k}^{n}\left(-\varsigma_{k}^{2}+\varsigma_{l}^{2}\right)}, \varsigma \neq 0,
\end{aligned}
$$

and $-1<\varsigma_{k}<1$

Now, set $\varsigma_{l}=l, l=1,2, \ldots, n$, in (3.4), we find a polynomial such that $Q_{2}^{(n-1)}(u)=\frac{(n+1) !}{n} \sum_{k=1}^{n} \frac{k}{n} \frac{(2 n) !}{(n-k) !(n+k) !}(-u)^{k-1}, n \geq 1, \forall k=1,2, \ldots$

Now, with the aid of (2.9), and (3.5) we find that

$$
Q_{2}^{(n-1)}(u)=\frac{(n+1) !}{n} P_{n-1}, n \geq 1
$$

Thus due to the relation (3.6) and the analysis given in [10, section-2, eqns. (2.3)-(2.8)], we may guess the curve of the trigonometric sum which has no non- zero zeros (in form of natural numbers) of Legendre function.

Again, put $\varsigma_{l}=\frac{1}{l}, \forall l=1,2, \ldots, n$, in (3.4), we find that

$$
R_{2}^{(n-1)}(u)=(-1)^{n-1} \prod_{l=1}^{n}-1\left(\frac{n^{2}-l^{2}}{n^{2} l^{2}}\right) \sum_{k=1}^{n} \frac{k \prod_{l=1}^{n}\left(\frac{l+1}{l}\right)(u)^{1 / k^{-1}}}{\prod_{l=1, l \neq k}^{n}\left(\frac{k^{2}-l^{2}}{k^{2} l^{2}}\right)}
$$


Then on solving (3.7) we easily obtain

$$
R_{2}^{(n-1)}(u)=\left(\frac{n+1}{n}\right) \sum_{k=1}^{n}\left(\frac{k}{n}\right)^{2 n-1} \frac{(2 n) !}{(n-k) !(n+k) !}(-u)^{k-1}
$$

where, $u_{k}=u^{-1 / k}, 0<u<1$.

Now we suppose that

$$
S_{2}^{(n-1)}(u)=u R_{2}^{(n-1)}(u), S_{2}^{(n-1)}(u)
$$

is many times differentiable, $n \geq 1$. (3.9)

Then we find the relation

$$
\left(u \frac{d}{d u}\right)^{2 n-2} S_{2}^{(n-1)}(u)=\frac{(n+1)}{(n)^{2 n-1}} H_{n-1}(u), n \geq 1 .
$$

The function $H_{n-1}(u)$ is defined in the equation (2.14).

From (3.10) we may guess the curve on which the distributions of nonzero real zeros lie between -1 to +1 of Legendre function.

\section{On the Infinite Divisibility}

Theorem-3 : The distributions, with the probability densities (1.8) and (1.9), are infinite divisible and thus (1.6) and (1.7) are also infinite divisible.

Proof : (A) : From (1.8), we write the equality (See Takano [10], p.3)

$$
\begin{aligned}
f_{1}(x)=\lambda_{1} \frac{\prod_{k=1}^{n}\left(z_{k}^{2}\right)}{\prod_{k=1}^{n}\left(x^{2}+z_{k}^{2}\right)}=\lambda_{1} \sum_{k=1}^{n} \frac{\prod_{l=1}^{n}\left(z_{l}^{2}\right)}{\prod_{l=1, l \neq k}^{n}\left(-z_{k}^{2}+z_{l}^{2}\right)\left(x^{2}+z_{k}^{2}\right)}, \\
-\infty<x<\infty, z_{k} \neq 0, \forall k=1,2, \ldots, n .
\end{aligned}
$$


Again, using the relation

$$
\frac{1}{x^{2}+z_{k}^{2}}=\int_{0}^{\infty} \frac{1}{\sqrt{\pi}(v)^{\alpha}} e^{-x^{2} / v} \sqrt{\pi e}^{-z_{k}^{2} / v}(v)^{\alpha-2} d v, \alpha>1
$$

and $\alpha \neq 2,3, \ldots, z_{k} \neq 0$, in (4.1) we find that

$f_{1}(x)=\int_{0}^{\infty} \frac{1}{\sqrt{\pi}(v)^{\alpha}} e^{-x^{2} / v} \sum_{k=1}^{n} \frac{\lambda_{1} \sqrt{\pi} \prod_{l==1}^{n}\left(z_{l}^{2}\right)}{\prod_{l=1, l \neq k}^{n}\left(-z_{k}^{2}+z_{l}^{2}\right)} e^{-z_{k}^{2} / v}(v)^{\alpha-2} d v, \alpha>1$

$\alpha>1$ and $\alpha \neq 2,3, \ldots, z_{k} \neq 0, \forall k=1,2, \ldots, n$.

Now, from (4.2) we set that

$$
g_{1}(v)=\lambda_{1} \sqrt{\pi} \sum_{k=1}^{n} \frac{\prod_{l==1}^{n}\left(z_{l}^{2}\right)}{\prod_{l=1, l \neq k}^{n}\left(-z_{k}^{2}+z_{l}^{2}\right)} e^{-z_{k}^{2} / v}(v)^{\alpha-2}
$$

$\alpha>1$ and $\alpha \neq 2,3, \ldots, z_{k} \neq 0, \forall k=1,2, \ldots, n, v>0$.

When $\mathrm{n}=1$, in (4.3), we have

$$
g_{1}(v)=\lambda_{1} \sqrt{\pi} z_{1}^{2} e^{-z_{1}^{2} / v}(v)^{\alpha-2}, z_{1} \neq 0, \alpha>1
$$

and $\alpha \neq 2,3, \ldots, v>0$

When $n=2$, we have

$g_{1}(v)=\lambda_{1} \sqrt{\pi}\left[\frac{z_{1}^{2}}{\left(-z_{1}^{2}+z_{2}^{2}\right)} e^{-z_{1}^{2} / v}+\frac{z_{2}^{2}}{\left(-z_{1}^{2}+z_{2}^{2}\right)} e^{-z_{2}^{2} / v}\right](v)^{\alpha-2}, z_{1}, z_{2} \neq 0, \alpha>1$

and $\alpha \neq 2,3, \ldots, v>0$ 
In the above results (4.3)-(4.5) the distribution $g_{1}(v)$ is of type normal distribution and has the finite variables $\left(z_{1}, \ldots, z_{n}\right)$ and is continuous to the variable $v$ so that it is continuous and partially discrete thus it is a mixing density function of normal distribution and it is positive for $\alpha>1$ and $\alpha \neq 2,3, \ldots, v>0$. Again the distribution with the density $g_{1}(v)$ is infinitely divisible and hence for that following relations hold:

$$
-\chi_{1}^{\prime}(s)=\chi_{1}(s) \int_{0}^{\infty} e^{-s t} k_{1}(t) d t
$$

where

$$
\chi_{1}(s) \int_{0}^{\infty} e^{-s v} g_{1}(v) d v
$$

The $k_{1}(t)$ is a non-negative bounded function given by

$$
k_{1}(t)=\lim _{R \rightarrow \infty} \frac{1}{2 \pi i} \int_{\xi-i R}^{\xi+i R} e^{t s}\left(-\frac{\chi_{1}^{\prime}(s)}{\chi_{1}(s)}\right) d s, \xi>0, t>0 .
$$

Then, make an appeal to the equations (4.4) and (4.7) we easily find that

$$
\chi_{1}(s)=\lambda_{1} \sqrt{\pi} z_{1}^{2} \sum_{m=0}^{\infty} \frac{\Gamma(\alpha-m-1)}{m !}\left(-z_{1}^{2}\right)^{m}(s)^{m-\alpha+1}, \alpha>1
$$

and $\alpha \neq 2,3, \ldots$

Then from (4.9) we have

$$
\chi_{1}^{\prime}(s)=-\lambda_{1} \sqrt{\pi} z_{1}^{2} \sum_{m=0}^{\infty} \frac{\Gamma(\alpha-m)}{m !}\left(-z_{1}^{2}\right)^{m}(s)^{m-\alpha}, \alpha>1
$$

and $\alpha \neq 2,3, \ldots(4.10)$

Thus from the equations (4.8), (4.9) and (4.10), we obtain

$$
k_{1}(t)=\lim _{R \rightarrow \infty} \frac{1}{2 \pi i} \int_{\xi-i R}^{\xi+i R} e^{t s}\left(\frac{\sum_{m=0}^{\infty} \frac{\Gamma(\alpha-m)}{m !}\left(-z_{1}^{2}\right)^{m}(s)^{m-\alpha}}{s \sum_{m=0}^{\infty} \frac{\Gamma(\alpha-m-1)}{m !}\left(-z_{1}^{2}\right)^{m}(s)^{m-\alpha}}\right) d s,
$$

$\alpha>1$ and $\alpha \neq 2,3, \ldots, \xi>0, t>0$ 
Check the following inequalities for all $m=0,1,2, \ldots$, and $\alpha \neq 2,3, \ldots$ $\alpha+m+1>\alpha+m \Rightarrow \Gamma(\alpha-m)>\Gamma(\alpha-m-1)$, and

$$
\alpha<\alpha+m+1 \Rightarrow \Gamma(\alpha-m)<\alpha \Gamma(\alpha-m-1)
$$

Then, from the inequalities given in (4.12) we easily obtain

$$
\frac{1}{s}<\left(\frac{\sum_{m=0}^{\infty} \frac{\Gamma(\alpha-m)}{m !}\left(-z_{1}^{2}\right)^{m}(s)^{m-\alpha}}{s \sum_{m=0}^{\infty} \frac{\Gamma(\alpha-m-1)}{m !}\left(-z_{1}^{2}\right)^{m}(s)^{m-\alpha}}\right)<\frac{\alpha}{s}
$$

Then, with the help of (4.11) and (4.13) we easily obtain

$$
0<k_{1}(t)<(\alpha-1), \alpha>1, \text { and } \alpha \neq 2,3, \ldots, \forall t \in(-\infty, \infty)
$$

(Since $k_{1}(t)$ is independent of $t$.)

Thus the relations (4.6)-(4.8) hold and $k_{1}(t)$ is non-negative for $\alpha>1$, and $\alpha \neq 2,3, \ldots, \forall t \in(-\infty, \infty)$ and thus the density function $g_{1}(v)$ is infinitely divisible (See also [3], [8], [9], [10] [11]) and therefore the distribution with density function (1.8) is also infinitely divisible.

Again, there exists

$$
\lim _{n \rightarrow \infty} f_{1}(x)=\lim _{n \rightarrow \infty} \lambda_{1} \frac{\prod_{k=1}^{n}\left(z_{k}^{2}\right)}{\prod_{k=1}^{n}\left(x^{2}+z_{k}^{2}\right)} \rightarrow \frac{\lambda_{1}}{\prod_{k=1}^{\infty}\left(1+\frac{x^{2}}{z_{k}^{2}}\right)}
$$

where $\gamma_{1}$ is an arbitrary constant, $-\infty<x<\infty$, and $z_{k} \neq 0, \forall k=1,2 \ldots$.

Therefore, the distribution with density (1.6) is also infinitely divisible.

The probability distribution with density (1.6) converges in the sense: (See, [7], [10])

$$
\Phi_{1}(t) \rightarrow \Psi_{1}(t)=\int_{-\infty}^{\infty} e^{i t x} \frac{\lambda_{1}}{\prod_{k=1}^{\infty}\left(1+\frac{x^{2}}{z_{k}^{2}}\right)} d x, \lambda_{1}
$$

is an arbitrary constant, $-\infty<t<\infty$, and $z_{k} \neq 0, \forall k=1,2 \ldots$

(B)- In the similar manner, from (1.8) we write the equality

$$
f_{2}(x)=\lambda_{2} \frac{\prod_{k=1}^{n}\left(1-\varsigma_{k}^{2}\right)}{\prod_{k=1}^{n}\left(x^{2}+\varsigma_{k}^{2}\right)}=\lambda_{2} \sum_{k=1}^{n} \frac{\prod_{t==1}^{n}\left(1-\varsigma_{k}^{2}\right)}{\prod_{l=1, l \neq k}^{n}\left(-\varsigma_{k}^{2}+\varsigma_{l}^{2}\right)\left(x^{2}+\varsigma_{k}^{2}\right)},
$$


$-\infty<x<\infty, \varsigma_{k} \neq 0$ and $-1<\varsigma_{k}<1, \forall k=1,2, \ldots$

Again, with the help of the relation given in (4.2) and (4.17) we find

$$
\begin{gathered}
f_{2}(x)=\int_{0}^{\infty} \frac{1}{\sqrt{\pi(v)^{\alpha}}} e^{-x^{2} / v} \sum_{k=1}^{n} \frac{\lambda_{2} \sqrt{\pi} \prod_{l==1}^{n}\left(1-\varsigma_{l}^{2}\right)}{\prod_{l=1, l \neq k}^{n}\left(-\varsigma_{k}^{2}+\varsigma_{l}^{2}\right)} e^{-\varsigma_{k}^{2} / v}(v)^{\alpha-2} d v, \alpha>1 \\
\text { and } \alpha \neq 2,3, \ldots, \varsigma_{k} \neq 0 \text { and }-1<\varsigma_{k}<1, \forall k=1,2, \ldots
\end{gathered}
$$

Now, from (4.18) we set that

$$
\begin{gathered}
g_{2}(v)=\lambda_{2} \sqrt{\pi} \sum_{k=1}^{n} \frac{\prod_{l==1}^{n}\left(1-\varsigma_{l}^{2}\right)}{\prod_{l=1, l \neq k}^{n}\left(-\varsigma_{k}^{2}+\varsigma_{l}^{2}\right)} e^{-\varsigma_{k}^{2} / v}(v)^{\alpha-2}, \\
\alpha>1 \text { and } \alpha \neq 2,3, \ldots, \varsigma_{k} \neq 0 \text { and }-1<\varsigma_{k}<1, \forall k=1,2, \ldots, v>0
\end{gathered}
$$

When $n=1$ in (4.19), we have

$$
g_{2}(v)=\lambda_{2} \sqrt{\pi}\left(1-\varsigma_{1}^{2}\right) e^{-\varsigma_{1}^{2} / v}(v)^{\alpha-2}, \varsigma_{1} \neq 0,-1<\varsigma_{1}<1, \alpha>1
$$

and $\alpha \neq 2,3, \ldots, v>0$

When $n=2$ in (4.19), we have

$$
g_{2}(v)=\lambda_{2} \sqrt{\pi}\left[\frac{1-\varsigma_{1}^{2}}{\left(-\varsigma_{1}^{2}+\varsigma_{2}^{2}\right)} e^{-\varsigma_{1}^{2} / v}+\frac{1-\varsigma_{1}^{2}}{\left(-\varsigma_{2}^{2}+\varsigma_{1}^{2}\right)} e^{-\varsigma_{2}^{2} / v}\right](v)^{\alpha-2}, \varsigma_{1}, \varsigma_{2} \neq 0
$$

$$
\text { and }-1<\varsigma_{1}<1,-1<\varsigma_{2}<1, \alpha>1 \text {, and } \alpha \neq 2,3, \ldots, v>0 \text {. }
$$


The above results (4.19)-(4.21) show that $\mathrm{g}_{2}(v)$ is a mixing probability density function of normal distribution and it is positive for $\alpha>1$ and $\alpha \neq 2,3, \ldots, v>0$. Again the distribution with the density $g_{2}(v)$ is infinitely divisible and for that following relations hold:

$$
-\chi_{2}^{\prime}(s)=\chi_{2}(s) \int_{0}^{\infty} e^{-s t} k_{2}(t) d t
$$

where

$$
\chi_{2}(s)=\int_{0}^{\infty} e^{-s v} g_{2}(v) d v
$$

The $k_{2}(t)$ is a non-negative bounded function and there exists

$$
k_{2}(t)=\lim _{R \rightarrow \infty} \frac{1}{2 \pi i} \int_{\xi-i R}^{\xi+i R} e^{t s}\left(\frac{\chi_{2}^{\prime}(s)}{\chi_{2}(s)}\right) d s, \xi>0, \forall t \in(-\infty, \infty) .
$$

From the techniques applied in (4.9)-(4.14), we examine and conclude that $k_{2}(t)$ is non-negative and bounded function for $\alpha>1$ and $\alpha \neq$ $2,3, \ldots, \forall t \in(-\infty, \infty)$ and thus the density function $g_{2}(v)$ is infinitely divisible (See also [3], [8], [9], [10] [11]) and therefore the distribution with density function (1.9) is also infinitely divisible.

Again there exists

$$
\lim _{n \rightarrow \infty} f_{2}(x)=\lim _{n \rightarrow \infty} \lambda_{2} \frac{\prod_{k=1}^{n}\left(1-\varsigma_{k}^{2}\right)}{\prod_{k=1}^{n}\left(x^{2}+\varsigma_{k}^{2}\right)} \rightarrow \frac{\lambda_{2}}{\prod_{k=1}^{\infty}\left(\frac{x^{2}+\varsigma_{k}^{2}}{1-\varsigma_{k}^{2}}\right)}
$$

where $\gamma_{2}$ is an arbitrary constant, $-\infty<x<\infty$, and $\varsigma_{k} \neq 0$ and $-1<$ $\varsigma_{k}<1, \forall k=1,2 \ldots$ Therefore, the distribution with density (1.7) is also infinitely divisible.

The probability distribution with density (1.7) converges in the sense: (See, [7], [10])

$$
\Phi_{2}(t) \rightarrow \Psi_{2}(t)=\int_{-\infty}^{\infty} e^{i t x} \frac{\lambda_{2}}{\prod_{k=1}^{\infty}\left(\frac{x^{2}+\varsigma_{k}^{2}}{1-\varsigma_{k}^{2}}\right)} d x, \lambda_{2}
$$


is an arbitrary constant, $-\infty<t<\infty$, and $\varsigma_{k} \neq 0,-1<\varsigma_{k}<1$, $\forall k=1,2, \ldots$

is an arbitrary constant, $-\infty<t<\infty$, and $\varsigma_{k} \neq 0,-1<\varsigma_{k}<1, \forall k=1,2 \ldots$

\section{Discussions}

Takano [10, section-2, eqn. (2.4)] has taken a polynomial

$$
h(u)=\frac{1}{n} \sum_{k=1}^{n}(-1)^{k} \frac{2 n !}{(n-k) !(n+k) !} u^{k}, \frac{d}{d u} h(u)=-P_{n-1}(u)
$$

Then, from (5.1) for $u=e^{i \theta},(0 \leq \theta \leq 2 \pi)$ and using following identities

$$
\sum_{k=1}^{n}(-1)^{k} \frac{2 n !}{(n-k) !(n+k) !} \cos k \theta=2^{2 n-1} \sin ^{2 n}\left(\frac{\theta}{2}\right)-\frac{1}{2}\left(\begin{array}{c}
2 n \\
n
\end{array}\right) \text {, }
$$

and

$$
\begin{aligned}
& \quad \sum_{k=1}^{n}(-1)^{k} \frac{2 n !}{(n-k) !(n+k) !} \sin k \theta=-\sin \theta \sum_{r=0}^{n-1} \frac{(2 n-2 r-2) !}{\{(n-r-1) !\}^{2}} \\
& 2^{2 r}\left(\sin \frac{\theta}{2}\right)^{2 r}
\end{aligned}
$$

Takano [10] has guessed the curve of trigonometric sum given in the equations (5.2) and(5.3).

Now consider a polynomial

$$
D_{1}^{(n)}(u)=\sum_{k=1}^{n}(-1)^{k} \frac{(2 n) !(n-1) !}{(n-k) !(n+k) !} u^{k}
$$


Then by equations (2.9), (2.10) and (5.4) we have

$$
\frac{d}{d u} D_{1}^{(n)}(u)=-(n) ! P_{n-1}(u)=-Q_{1}^{(n-1)}(u), n \geq 1 .
$$

Now in (5.4) take $u=e^{i \theta},(0 \leq \theta \leq 2 \pi)$ and use the identities (5.2) and (5.3) and suppose that

$$
\begin{aligned}
& D_{1}^{(n)}\left(e^{i \theta}\right)=U(\theta)+i V(\theta) \text { we get } \\
& U(\theta)=\frac{1}{n}\left[2^{2 n-1}(n) ! \sin ^{2 n}\left(\frac{\theta}{2}\right)-\frac{1}{2} \frac{(2 n) !}{(n) !}\right]
\end{aligned}
$$

and

$$
\mathrm{V}(\theta)=-(n-1) ! \sin \theta \sum_{r=0}^{n-1} \frac{(2 n-2 r-2) !}{\{(n-r-1) !\}^{2}} 2^{2 r}\left(\sin \frac{\theta}{2}\right)^{2 r}, n \geq 1
$$

Thus from right hand side of (5.6) we may guess the curve whose slope has non-zero zeros of Bessel function.

Further put $u=e^{i m \theta},\left(0 \leq \theta \leq \frac{2 \pi}{m}, m>k\right)$ in (2.14) we get

$$
\begin{gathered}
H_{n-1}\left(e^{i m \theta}\right)=-\frac{1}{n}\left[\sum_{k=1}^{n} \frac{(2 n) !(-1)^{k} k}{(n-k) !(n+k) !} \cos \left(\frac{m}{k} \theta\right)\right. \\
\left.+i \sum_{k=1}^{n} \frac{(2 n) !(-1)^{k} k}{(n-k) !(n+k) !} \sin \left(\frac{m}{k} \theta\right)\right], n \geq 1,(5.6)
\end{gathered}
$$

At $\theta=0$ from (5.7) we have

$$
H_{n-1}\left(e^{i m \theta}\right)=\frac{(2 n-2) !}{(n-1) !(n) !}, n \geq 1
$$

Thus using the relations (2.15) and (3.10) in (5.7) and (5.8) respectively we may find the curves which have the non-zero zeros (in rational form) of Bessel and Legendre functions. Due to lack of space we omit them.

\section{References}

[1] M. Abramowitz and I. A. Stegun, Handbook of Mathematical Functions, Dover Publication, New York, (1970).

[2] L. Bondesson, On the infinite divisibility of the half-Cauchy and other decreasing densities and probability functions on the non-negative line, Scand. Acturial J., pp. 225-247, (1985). 
[3] M. J. Goovaerts, L. D Hooge and N. De, Pril, On the infinite divisibility of the product of two $\Gamma$-distributed stochastically variables, Applied Mathematics and Computation, 3, pp. 127-135, (1977).

[4] H. Hochstadt, The Functions of Mathematical Physics, New York, Dover, (1986).

[5] D. H. Kelkar, Infinite divisibility and variance mixtures of the normal distribution, Ann. Math. Statistic. 42, pp. 802-808, (1971).

[6] H. Kumar and S. Srivastava, On some sequence of integrals and their applications, Bull. Cal. Math. Soc. 100 (5), pp. 563-572, (2008).

[7] K. Sato, Class L of multivariate distributions and its sub classes, J. Multivaria. Anal. 10 (1980), pp. 207-232, (1980).

[8] F. W. Steutel, Preservation of infinite divisibility under mixing and related topics, Math. Center, Tract. Amsterdam, 33, (1970).

[9] K. Takano, On a family of polynomials with zeros outside the unit disk, Int. J. Comput. Num. Anal. Appl. 1 (4), pp. 369-382, (2002).

[10] K. Takano, On the infinite divisibility of normed conjugate product of Gamma function, Proc. of 4th Int. Conf. SSFA, (4), pp. 1-8, (2003).

[11] O. Thorin, On the infinite divisibility of the Pareto distribution, Scand. Acturial. J. pp. 31-40, (1977).

\section{Hemant Kumar}

Department of Mathematics, D. A-V. P. G., College, Kanpur, U. P.

India

e-mail : palhemant2007@rediffmail.com

\section{A. Pathan}

Department of Mathematics, University of Botswana, Private Bag 0022, Gaborone, Botswana

e-mail : mapathan@gmail.com 
182 Hemant Kumar, M. A. Pathan and R. C. Singh Chandel

and

R. C. Singh Chandel

Department of Mathematics,

D. V. P. G., College,

Orai, U. P.,

India

e-mail : rcschandel@yahoo.co.in 